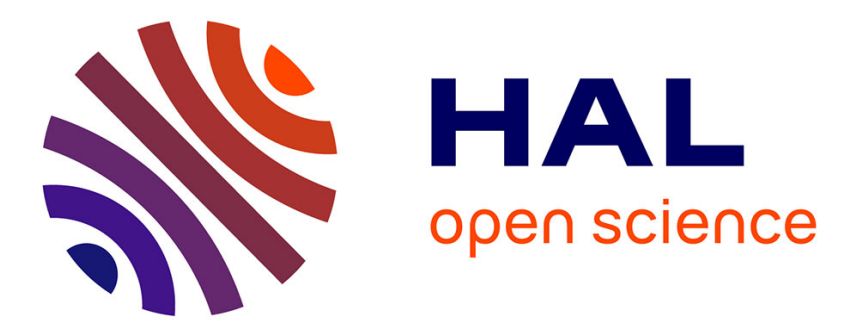

\title{
Period-infinity periodic motions, chaos, and spatial coherence in a 10 degree of freedom impact oscillator
}

\author{
J P Cusumano, B.-y P Bai
}

\section{To cite this version:}

J P Cusumano, B.-y P Bai. Period-infinity periodic motions, chaos, and spatial coherence in a 10 degree of freedom impact oscillator. Chaos, Solitons \& Fractals, 1993, 3, pp.515 - 535. 10.1016/09600779(93)90003-J . hal-01509343

\section{HAL Id: hal-01509343 \\ https://hal.science/hal-01509343}

Submitted on 17 Apr 2017

HAL is a multi-disciplinary open access archive for the deposit and dissemination of scientific research documents, whether they are published or not. The documents may come from teaching and research institutions in France or abroad, or from public or private research centers.
L'archive ouverte pluridisciplinaire HAL, est destinée au dépôt et à la diffusion de documents scientifiques de niveau recherche, publiés ou non, émanant des établissements d'enseignement et de recherche français ou étrangers, des laboratoires publics ou privés. 


\title{
Period-infinity Periodic Motions, Chaos, and Spatial Coherence in a 10 Degree of Freedom Impact Oscillator
}

\author{
J. P. CUSUMANO and B.-Y. BAI \\ Department of Engineering Science \& Mechanics, The Pennsylvania State University. University Park, PA 16802. \\ USA
}

\begin{abstract}
The numerical study of a 10 degree of freedom impact oscillator is presented. The mathematical model consists of a linear array of masses in which each mass is connected to its nearest neighbor by identical springs and dashpots. One end mass is attached to a rigid support while the other is free to impact a sinusoidally vibrating rigid table. Bifurcation diagrams based on the impact Poincaré map are obtained over the entire range of natural frequencies, taking the table frequency as the bifurcation parameter. The diagrams reveal many chautic bands as well as a wide variety of period $(n, m)\left(P_{n, m}\right)$ orbits, where $n$ is the period with respect to the impact Poincaré map and $m$ is the number of table periods. Perhaps the most interesting solutions are $P_{x, m}$ solutions (where $m$ is finite). These arise from sticking events in which the time between impacts approaches zero in finite time. The convergence properties of the impact time sequence during a sticking event is shown to be controlled by the coefficient of restitution of the impact law. The spatial complexity of the motions is studied by finding proper orthogonal modes for various steady states, and the number of excited degrees of freedom is estimated by finding the number of modes needed to exceed $99 \%$ of the signal power. These results are compared to the predictions of fractal dimension theory.
\end{abstract}

\section{INTRODUCTION}

Impact oscillators have furnished a useful mechanical paradigm for discrete-time nonlinear dynamical systems, and have been the subject of many recent investigations, both theoretical [1-6], and experimental [7-11]. In engineering systems, impact is sometimes an essential part of the design, as in impact printers [12]; but, more often, it is the source of unwanted wear and noise, as in the dynamics of structures with joint clearances, or in gear systems. Whereas most previous studies of the nonlinear dynamics of impact oscillators have involved systems with, either literally or effectively, one degree of freedom, flexibility effects should be expected to play an important role in applications. Furthermore, from the elementary theory of impact, one anticipates that many degrees of freedom will be excited by impacts on flexible structures. Thus, flexible impact oscillators appear to be an ideal test bed with which to explore ideas of dimensionality and spatial complexity in the dynamics of solids and structures.

In this paper, we present the results of a numerical study of a 10 degree of freedom impact oscillator. The system is a linear array of masses in which each mass is connected to its nearest neighbor by identical linear springs and dashpots and in which one end mass is free to impact a sinusoidally vibrating rigid table. Since all of the nonlinearity is contained in the impact boundary condition, analytical solutions are available for motions between impacts. These solutions are used to construct the implicit 20-dimensional Poincaré map defined by the impact, and a computer code based on this map is used to obtain our results.

In Scction 2, we describe the physical system, its continuous-time mathematical model, and the associated impact-based Poincaré map. In Section 3, details of the computer 
algorithm used in our numerical experiments are presented, and the properties of sticking events are described: it is shown that the impact time sequence during a sticking event is controlled by the coefficient of restitution of the impact law and that sticking occurs in finite time. A modified algorithm is described which allows the simulations to proceed across sticking events. The bifurcation structure of the system is presented in Section 4 , and nature of the various solutions are described with the help of Poincaré sections, including chaotic as well as a wide variety of period- $(n, m)\left(P_{n, m}\right)$ solutions, where $n$ is the period with respect to the impact Poincare map and $m$ is the number of table periods. In particular, it is shown that sticking events can result in $P_{x, m}$ solutions (where $m$ is finite). In Section 5, we give a brief introduction to the proper orthogonal decomposition method for the study of spatial complexity, and we describe its application to our problem. The results of the proper orthogonal decomposition analysis are then presented, along with fractal dimension estimates for various steady states, and results are compared to the predictions of dimension theory. Section 6 is reserved for a summary and discussion of our results.

\section{THE MATHEMATICAL MODEL AND METHOD OF SOLUTION}

The object of this study is the system shown in Fig. 1. It consists of 10 identical masses of mass $m$ connected to nearest neighbors by identical linear springs and dashpots with constants $k$, and $c$, respectively. One end mass is connected to a rigid wall, again with a linear spring and dashpot, whereas the other end mass is free to impact on a sinusoidally moving rigid table. The entire system is constrained to permit only horizontal motion, with coordinates $x_{1}$ through $x_{10}$ defined in an obvious manner as in the figure. Between impacts, the system is completely linear, and thus can be represented by a dimensionless system of equations in the form

$$
\ddot{\mathbf{x}}+\gamma \mathbf{A} \dot{\mathbf{x}}+\mathbf{A x}=\mathbf{0}
$$

where $\mathbf{x}^{\mathrm{T}}=\left(x_{1}, x_{2}, \ldots, x_{10}\right), \gamma=c \sqrt{ }(\mathrm{km})$ is a dimensionless damping parameter, and the overdot denotes the derivative with respect to the dimensionless time $t$, which is related to the dimensional time $\bar{t}$ by $t=(\sqrt{ }(k / m)) \bar{t}$. A is a $10 \times 10$ banded matrix given by:

$$
\mathbf{A}=\left[\begin{array}{rrrrrrr}
2 & -1 & 0 & \ldots & 0 & 0 & 0 \\
-1 & 2 & -1 & \ldots & 0 & 0 & 0 \\
0 & -1 & 2 & \ldots & 0 & 0 & 0 \\
\vdots & \vdots & \vdots & & \vdots & \vdots & \vdots \\
0 & 0 & 0 & \ldots & 2 & -1 & 0 \\
0 & 0 & 0 & \ldots & -1 & 2 & -1 \\
0 & 0 & 0 & \ldots & 0 & -1 & 1
\end{array}\right] .
$$

Using the modal matrix $\boldsymbol{\Phi}$ (normalized so that $\boldsymbol{\Phi}^{\mathrm{T}} \boldsymbol{\Phi}=\mathbf{I}$ ), we transform the system to modal coordinates $\mathbf{q}^{\mathrm{T}}=\left(q_{1}, q_{2}, \ldots, q_{10}\right)$ by

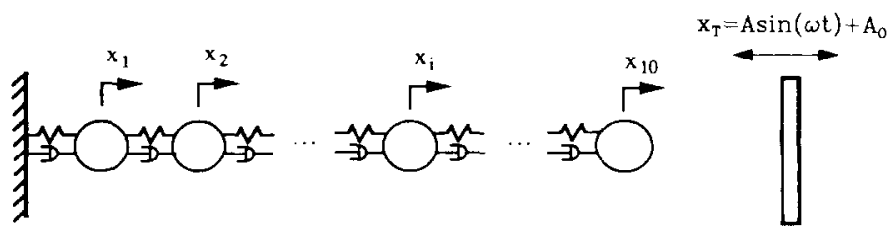

Fig. 1. Configuration of the 10 degree of freedom impact oscillator. 


$$
\mathbf{x}=\boldsymbol{\Phi q}
$$

Equation (1) then becomes

$$
\ddot{\mathbf{q}}+\gamma \mathbf{\Omega} \dot{\mathbf{q}}+\mathbf{\Omega q}=\mathbf{0}
$$

$\boldsymbol{\Omega}=\operatorname{diag}\left(\omega_{i}\right), i=1,2, \ldots, 10$, is the diagonal matrix of natural frequencies. That is, one obtains 10 uncoupled modal equations

$$
\ddot{q}+2 \zeta_{i} \omega_{i} \dot{q}_{i}+\omega_{i}^{2} q=0 \quad(i=1,2, \ldots, 10),
$$

in which the modal damping ratios $\zeta_{i}$ are, as usual, defined by $\zeta_{i}=\left(\gamma \omega_{i}\right) / 2$. The solution to the system of equations (5) is then simply

$$
q_{i}=\mathrm{c}^{-\zeta_{i} \omega_{i} t}\left(a_{i} \cos \bar{\omega}_{i} t+b_{i} \sin \bar{\omega}_{i} t\right),
$$

where $\bar{\omega}_{i}=\omega_{i} \vee\left(1-\zeta_{i}^{2}\right)$ is the damped natural frequency, and the constants $a_{i}$ and $b_{i}$ are determined from the initial data at time $t_{0}$ as

$$
a_{i}=q_{i}\left(t_{0}\right) ; \text { and } b_{i}=\frac{\dot{q}_{i}\left(t_{0}\right)+\zeta_{i} w_{i} q_{i}\left(t_{0}\right)}{\bar{\omega}_{i}} .
$$

Given the modal solutions (6), the solution in spatial coordinates is then obtained from the transformation (3). (The natural frequencies for the system between impacts are presented in Appendix A. We defer display of the normal modes until the discussion of spatial coherence in Section 5.)

The system evolves according to the solutions (6) until an impact occurs. That is, until

$$
x_{10}\left(t_{n}\right)=x_{\mathrm{T}}\left(t_{n}\right) .
$$

where $t_{n}$ is the time of the $n$th impact and the table motion is given by $x_{\mathrm{T}}(t)=A \sin \omega t$ $+A_{0}$. Equation (8) defines a scalar-valued function of time, the first root of which gives the impact time. Employing a simple impact theory in which the impact is assumed to occur instantaneously, just after the $n$th impact one has

$$
\begin{aligned}
x_{i}\left(t_{n}^{+}\right) & =x_{i}\left(t_{n}^{-}\right)=x_{i}\left(t_{n}\right) \quad(i=1,2, \ldots, 10) \\
v_{i}\left(t_{n}^{+}\right) & =v_{i}\left(t_{n}^{-}\right)=v_{i}\left(t_{n}\right) \quad(i=1,2, \ldots, 9) \\
v_{10}\left(t_{n}^{+}\right) & =\alpha\left(v_{\mathrm{T}}\left(t_{n}\right)-v_{10}\left(t_{n}^{-}\right)\right)+v_{\mathrm{T}}\left(t_{n}\right)
\end{aligned}
$$

where $v_{i}$ denotes the velocity of the $i$ th mass, and plus and minus superscripts indicate the right and left hand limits as $t \rightarrow t_{n}$, respectively. In the impact law (9.3), $\alpha$ is the coefficient of restitution, and we use the fact that $v_{\mathrm{T}}\left(t_{n}^{+}\right)=v_{\mathrm{T}}\left(t_{n}^{-}\right)$. Relations (9) give new initial conditions at time $t_{n}$, which are then used to generate the solution for the system up to the next impact, via equations (3), (6), and (7).

Thus, the dynamics of the system is completely determined by the state just after each impact, and one need only consider the 20-dimensional Poincaré map defined by the impact condition, $\mathscr{P}: \Sigma \rightarrow \Sigma$, where the cross section $\Sigma$ is given by

$$
\Sigma=\left\{(\mathbf{x}, \mathbf{v}, t) \in \mathbb{R}^{10} \times \mathbb{R}^{10} \times S^{1} \mid \hat{\mathbf{e}}_{10} \cdot \mathbf{x}=x_{\mathrm{T}}, \hat{\mathbf{e}}_{10} \cdot \mathbf{v}>v_{\mathrm{T}}\right\},
$$

and where it is understood that $t$ is taken modulo $2 \pi / \omega$ (see Fig. 2). Let $\left\{\hat{\mathbf{e}}_{i}\right\}_{i=1}^{10}$ denote the standard basis, so that $\mathbf{x} \cdot \hat{\mathbf{e}}_{i}$ is the projection onto the $i$ th component of $\mathbf{x}$. Then, using the impact conditions ( 8$)$ and (10), $\mathscr{P}$ can be written as:

$$
\begin{aligned}
& x_{i, n+1}=\widehat{\mathbf{e}}_{i} \cdot \boldsymbol{\Phi} \mathbf{q}\left(t_{n}+\Delta t_{n} ; \mathbf{x}_{n}, \mathbf{v}_{n}\right) \quad(i=1,2, \ldots, 9) \\
& v_{i, n+1}=\widehat{\mathbf{e}}_{i} \cdot \boldsymbol{\Phi} \dot{\mathbf{q}}\left(t_{n}+\Delta t_{n} ; \mathbf{x}_{n}, \mathbf{v}_{n}\right) \quad(i=1,2, \ldots, 9)
\end{aligned}
$$




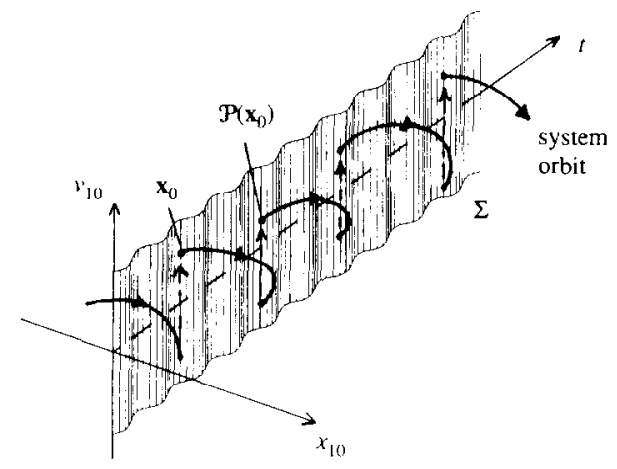

Fig. 2. Diagram showing the relation of the continuous-time system to the Poincare map defined by equations $(10-12)$.

$$
\begin{aligned}
v_{10, n+1} & =(1+\alpha) v_{\mathrm{T}}\left(t_{n}+\Delta t_{n}\right)-\alpha \widehat{\mathbf{e}}_{10} \cdot \mathbf{\Phi} \dot{\mathbf{q}}\left(t_{n}+\Delta t_{n} ; \mathbf{x}_{n}, \mathbf{v}_{n}\right) \\
t_{n+1} & =t_{n}+\Delta t_{n}
\end{aligned}
$$

where $\Delta t_{n}=\Delta t_{n}\left(\mathbf{x}_{n}, \mathbf{v}_{n}, t_{n}\right)$ is determined by using equation (8) to find the time of the $(n+1)$ th impact. Using the same notation as equations $(11)$, equation (8) becomes

$$
D\left(\Delta t_{n}\right) \equiv \hat{\mathbf{e}}_{10} \cdot \mathbf{\Phi q}\left(t_{n}+\Delta t_{n} ; \mathbf{x}_{n}, \mathbf{v}_{n}\right)-A \sin \left(t_{n}+\Delta t_{n}\right)-A_{0}=0 .
$$

The functional dependance of $\mathbf{q}$ and $\dot{\mathbf{q}}$ on the state at the $n$th impact, $\left(\mathbf{x}_{n}, \mathbf{v}_{n}\right)$, comes from the evaluation of the constants in equations (7) with $t_{0}=t_{n}$, and from the transformation (3).

\section{NUMERICAL IMPLEMENTATION: STICKING EVENTS}

A simulation program written in double precision Fortran was used to implement the algorithm defined by equations (11) and (12). The program uses a standard root-finding algorithm based on Newton's method [13] to solve the latter equation for $\Delta t_{n}$. Our code was verified by comparing the natural frequencics obtained from numerical impulseresponse tests to the natural frequencies obtained by solving the eigenvalue problem (see Appendix A): the results were identical to the numerical precision of the simulations.

In order to obtain the numerical data which was the aim of this study, a technical problem with mathematical and physical implications had to be addressed. A naive implementation of the algorithm defined by equations (11) and (12) is very efficient as long as the time between impacts is not too small. However, in certain orbits, sticking events can occur in which the $\Delta t_{n}$ rapidly decrease by many orders of magnitude: the calculations drop into the round-off-induced numerical noise floor, resulting in spurious, noisy bounces which decay no further (with a $\Delta t$ of the order of $10^{-6}-10^{-7}$ ). The problem is not simply one of limited precision, however: even with infinite precision, these motions kill the algorithm, since for all practical purposes the code can never get through the event, in a kind of numerical version of Zeno's paradox.

The most natural way to compensate for this is to modify the basic algorithm to assume that values of $\Delta t_{n}$ below some threshold value indicate that the tip mass has 'stuck' to the moving table (i.e. $v_{10}-v_{\mathrm{T}} \equiv 0$ ). However, the main question is whether or not these sticking events actually occur: that is, whether or not $\sum\left\{\Delta t_{n}\right\}<\infty$ near a sticking event. To explore this further, define the impact time ratio 


$$
\lambda_{n}=\frac{\Delta t_{n}}{\Delta t_{n-1}}
$$

In Fig. 3, we plot $\lambda_{n}$ vs $n$ during a sticking event. As shown in the figure, the $\lambda_{n}$ converge to the coefficient of restitution, right up to the limits of precision of the calculations. (A spurious divergence in Fig. 3 occurs after about 80 impacts. Assuming an initial time step of size $O(1)$, this divergence occurs when $\Delta t_{n}<(0.85)^{80}=O\left(10^{-6}\right)$, i.e. within the noise floor.) Hence, by the ratio test, the sum of time intervals converges, and therefore the tip mass sticks to the table in finite time. Thus, sticking events are an essential aspect of the dynamics of impact oscillators, not merely ad hoc assumptions needed to make simulations feasible.

Following these observations, the minimum time step $\Delta t_{\min }$ was set at $10^{-6}$ : should the root-finder compute a value of $\Delta t_{n}<\Delta t_{\min }$, the tip mass is assumed to be constrained to lie on the moving table. Thus, the impacting system of Section 2 is replaced by a simple, non-impacting, 9 degree of freedom linear oscillator driven by a moving support. The time-step error $\varepsilon$ that results from this approximation is

$$
\varepsilon=\sum_{m=0}^{\infty} \Delta t_{n+m}=\sum_{m=0}^{\infty} \alpha^{m} \Delta t_{n} \leqslant\left(\Delta t_{\min }\right) \sum_{m=0}^{\infty} \alpha^{m}=\frac{\Delta t_{\min }}{1-\alpha} .
$$

For this work, $\alpha=0.85$, so the absolute precision of our time-step calculations was on the order of $7 \times 10^{-6}$. As we discuss in Section 5, analysis of the Poincaré section data showed that the long-time cumulative accuracy of our time-step calculations was of the order of $3 \times 10^{-4}$. By comparison, the maximum time steps were of the order of several table periods (i.e. with an order of magnitude of around 10), thus indicating a dynamic range for our time-step calculations of $70-90 \mathrm{~dB}$, comparable to experimental observations possible under ideal circumstances with $12-14$ bit data acquisition.

The reduced system (with the end mass stuck to the table) is easily derived by deleting the 10 th row of equation (1), and setting $x_{10} \equiv x_{\mathrm{T}}=A \sin \omega t+A_{0}$ : the reaction force $F_{\mathrm{R}}$ between $x_{10}$ and the table is found to be

$$
F_{\mathrm{R}}=\ddot{x}_{\mathrm{T}}+\gamma\left(\dot{x}_{\mathrm{T}}-\dot{x}_{9}\right)+\left(x_{\mathrm{T}}-x_{9}\right)
$$

where $x_{9}$ is determined from the solution of the constrained system. The root-finder is then used to solve for the time when $F_{\mathrm{R}}$ passes through zero (decreasing), thus indicating that tip and the table lose contact and the system is again governed by equations (11) and (12).

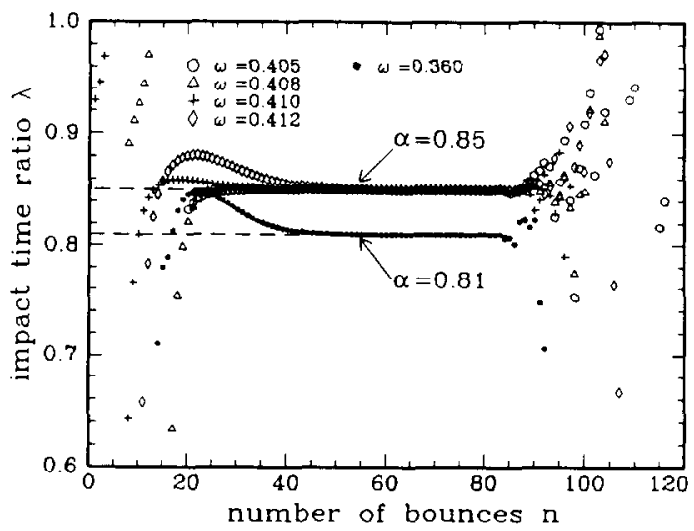

Fig. 3. Limiting behavior of impact time ratio during a sticking event. The ratios converge to the coefficient of restitution indicating that sticking occurs in finite time. 
It is perhaps not too surprising that the time-step ratio $\lambda_{n}$ converges to the coefficient of restitution. The limiting behavior during sticking events is thus identical to that seen in the elementary problem of a ball bouncing on a stationary floor [14]: when the time between impacts is very small in our system, the table and all but the end mass are essentially stationary. That is, the system behaves like a single degree of freedom impact oscillator during a sticking event. Mathematically, the problem of sticking arises from a degeneracy in the Poincaré map $\mathscr{P}$ [equations (11) and (12)]. The section $\Sigma$ is indeed global, however, it is not closed under the action of $\mathscr{P}$ : there exist initial conditions $\mathbf{X}_{0} \equiv\left(\mathbf{x}_{0}, \mathbf{v}_{0}, t_{0}\right) \in \Sigma$ such that $\mathscr{P}^{n}\left(\mathbf{X}_{0}\right) \in \Sigma$ for all $n<\infty$, but $\lim _{n \rightarrow \infty} \mathscr{P}^{n}\left(\mathbf{X}_{0}\right) \notin \Sigma$ since $\widehat{\mathbf{e}}_{10} \cdot \mathbf{v}_{n} \rightarrow v_{\mathrm{T}}$.

\section{BIFURCATION STRUCTURE}

Bifurcation diagrams were constructed for the system using the table frequency $\omega$ as the bifurcation parameter. Unless otherwise noted, system parameters used in the simulations were as follows: the coefficient of restitution $\alpha=0.85$; the table amplitude $A=0.10$; the table offset $A_{0}=0$; and the damping parameter $\gamma=0.1$. The algorithm used was capable only of following stable solutions: after allowing transients to die off by skipping the first 700 impacts, data from the next 100 impacts was stored for later display (in several test cases, it was found that skipping 1400 impacts before sampling left the results unchanged, so the lower number was used). The maximum value of $\Delta \omega$ was 0.002 : again, it was found in several test cases that doubling the resolution did not significantly change the results (more discussion about the stability of our results with respect to changes in $\Delta \omega$ can be found below). Data from the steady-state taken at $\omega=\omega_{m}$ was used to start the computations for $\omega=\omega_{m+1}$. The entire numerical experiment was started by releasing the system from rest in the shape of the first mode with unit amplitude. Tests done by releasing the system with modal amplitudes of 0.1 and 0.5 lead to the same results: however, given the large size of the phase space and time-consuming nature of the computations, it was not possible to search effectively for disconnected branches of solutions.

To cover the entire range of natural frequencies, we take $0.14 \leqslant \omega \leqslant 2.0$. The resulting bifurcation diagram is shown in Fig. 4, which plots the steady-state relative tip velocity $v_{1 \in l} \equiv v_{10}-v_{T}$ just after impact against $w$, along with labels indicating the type of response (the way in which the response type was determined is discussed below). Various types of period- $(n, m)\left(P_{n, m}\right)$ orbits are labelled, where $n$ is the period with respect to the number of table impacts and $m$ is the period with respect to the number of table periods. In addition, many chaotic bands are visible (labelled by $C_{n}$ ). That these labels by no means exhaust the range of responses is demonstrated in the blown-up portions of Fig. 4 which are presented in Fig. 5. Figures 4 and 5 demonstrate the sensitivity of the system as $\omega$ ranges over certain values: this is not just a computational artifact, since the overall structure of the bifurcation diagrams in both figures are the same, even though $\Delta \omega$ is $2.0 \times 10^{-3}$ in Fig. 4 and $2 \times 10^{-4}$ for Fig. 5 .

Figure 6(a) displays the same data as Fig. 4, however, following Geist and Lauderborn [15], we use the energy $E$ (dimensionless in our case) to characterize the system just after impact, where

$$
E=\frac{1}{2} \sum_{i=1}^{10} v_{i}^{2}+\frac{1}{2} x_{1}^{2}+\frac{1}{2} \sum_{i=2}^{10}\left(x_{i}-x_{i-1}\right)^{2} .
$$

Resonance-like peaks are clearly visible at two low frequencies $(\omega \approx 0.202$ and $\omega \approx 0.408)$ : note that the peaks do not occur at natural frequencies of the non-impacting system. At 


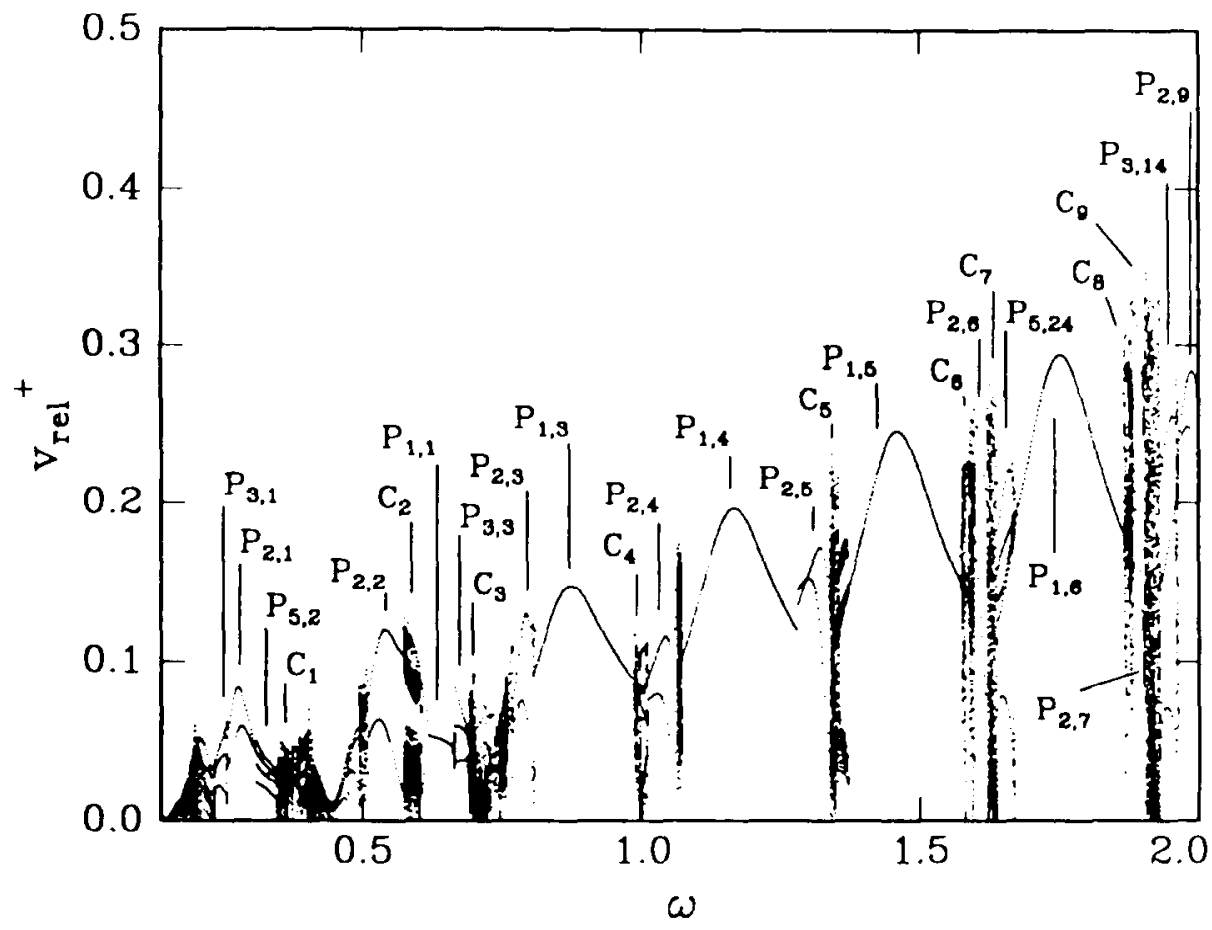

Fig. 1. Bifurcation diagram showing relative tip velocity just after impact: computed with a fixed table amplitude $\mathrm{A}=0.10$.

higher frequencies, no resonant peaks are observed, and the general trend is for increasingly more energetic motions: this is due to the fact that the diagrams of Figs 4,5 , and $6($ a) were generated with constant table amplitude $A$. Using equations (9) and (16), it is not hard to show that the change in energy across an impact is given by

$$
\Delta E=E^{+}-E^{-}=\frac{1}{2}\left[2(1+\alpha) v_{\mathrm{T}}\left|v_{\mathrm{rel}}^{-}\right|+\left(\alpha^{2}-1\right)\left(v_{\mathrm{rel}}^{-}\right)^{2}\right],
$$

which can be rearranged to give the bound:

$$
\Delta E=v_{\mathrm{T}}^{2}\left[(1+\alpha) \frac{\left|v_{\mathrm{rel}}^{-}\right|}{v_{\mathrm{T}}}+O\left(\left(\frac{v_{\mathrm{rel}}^{-}}{v_{\mathrm{T}}}\right)^{2}\right)\right] \leqslant v_{\mathrm{T}}^{2}(1+\alpha) M,
$$

where, for small amplitude oscillations (in which, just before impact, $\left.\left|v_{\text {rel }}^{-}\right| \ll\left|v_{\mathrm{T}}\right|\right), M \approx$ $\sup \left(\left|v_{\text {rel }}^{-}\right| / v_{\mathrm{T}}\right)$. Thus energy input is controlled by the table velocity, and a better measure of input amplitude is given by the velocity amplitude $\omega A$. A 'constant small input' bifurcation diagram obtained by holding $\omega A$ constant is shown in Fig. $6(\mathrm{~b})$. In this diagram, the resonances are more clearly visible, and the energy level remains roughly constant away from the resonances, confirming the analysis of equation (18). In addition, the response for frequencies about $\omega>\omega_{3}$ displays a periodic structure only hinted at in Figs 4 and 6(a).

Solutions were characterized using time series, impact-based and stroboscopic Poincaré sections, and power spectra (in Section 5, we also use spatial correlation and fractal dimension estimates). Continuous-time trajectories were obtained by interpolating between impact data using the solutions (6) and equation (3). Stroboscopic Poincaré sections were obtained by sampling the continuous time data at zero phase of the table oscillation $(\phi=\bmod (\omega t, 2 \pi)=0)$. In Figs 7 and 8 , results for a typical $P_{1.3}$ orbit are shown. The time 

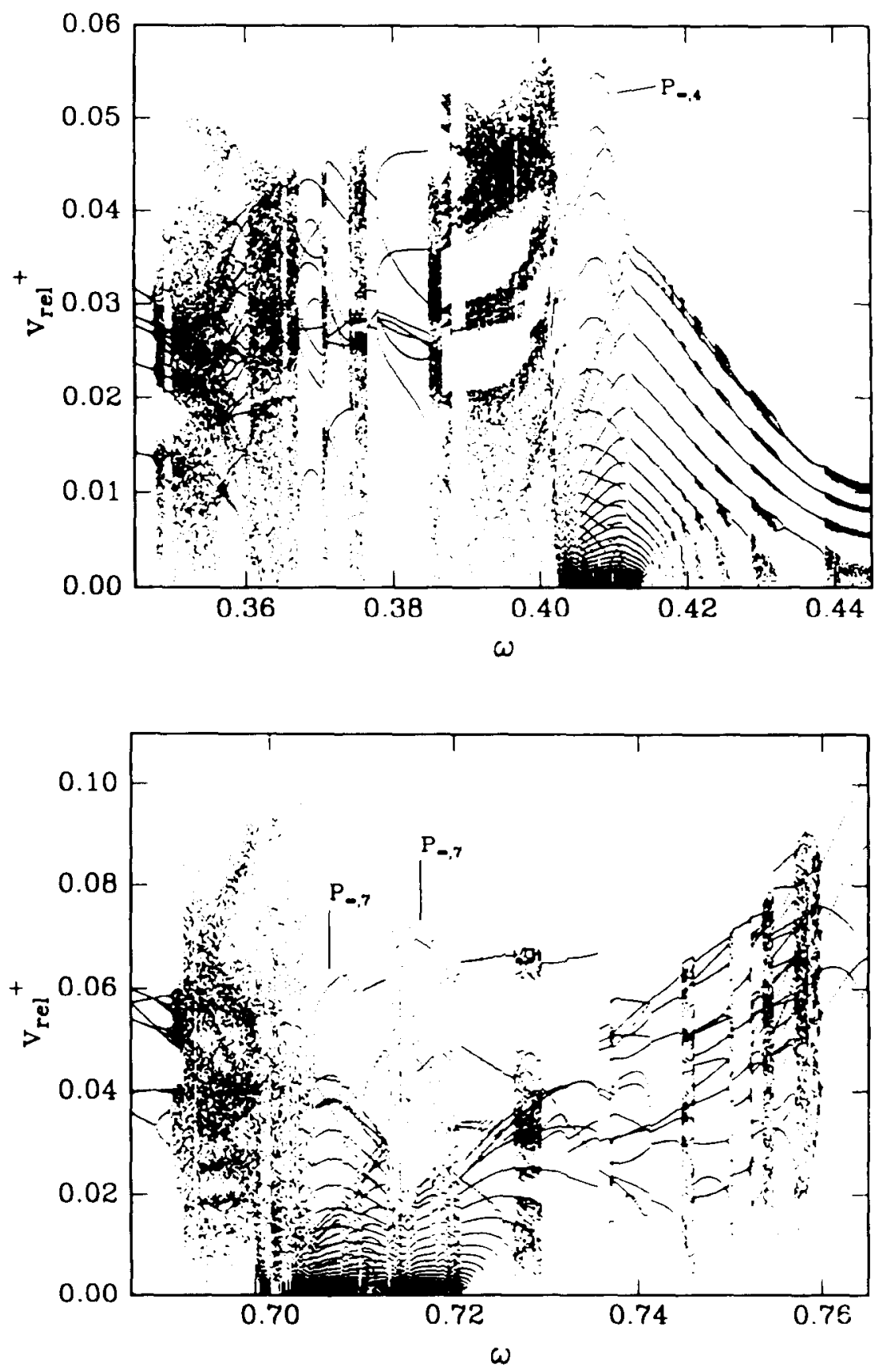

Fig. 5. Blown-up portions of bifurcation diagram of Fig. 4. Three different sticking motions are labelled in the diagrams.

series and power spectrum of Fig. 7, and the stroboscopic Poincaré section of Fig. 8 (bottom) all show that the orbit is period-3 with respect to the table motion, whereas the impact Poincaré section of Fig. 8 (top) shows that the orbit is period-1 with respect to the impact map of equations (11). In this manner, the character of the various solutions in Figs 4 were obtained. Regular motions can contain sticking events: an example of such a motion 
(a)

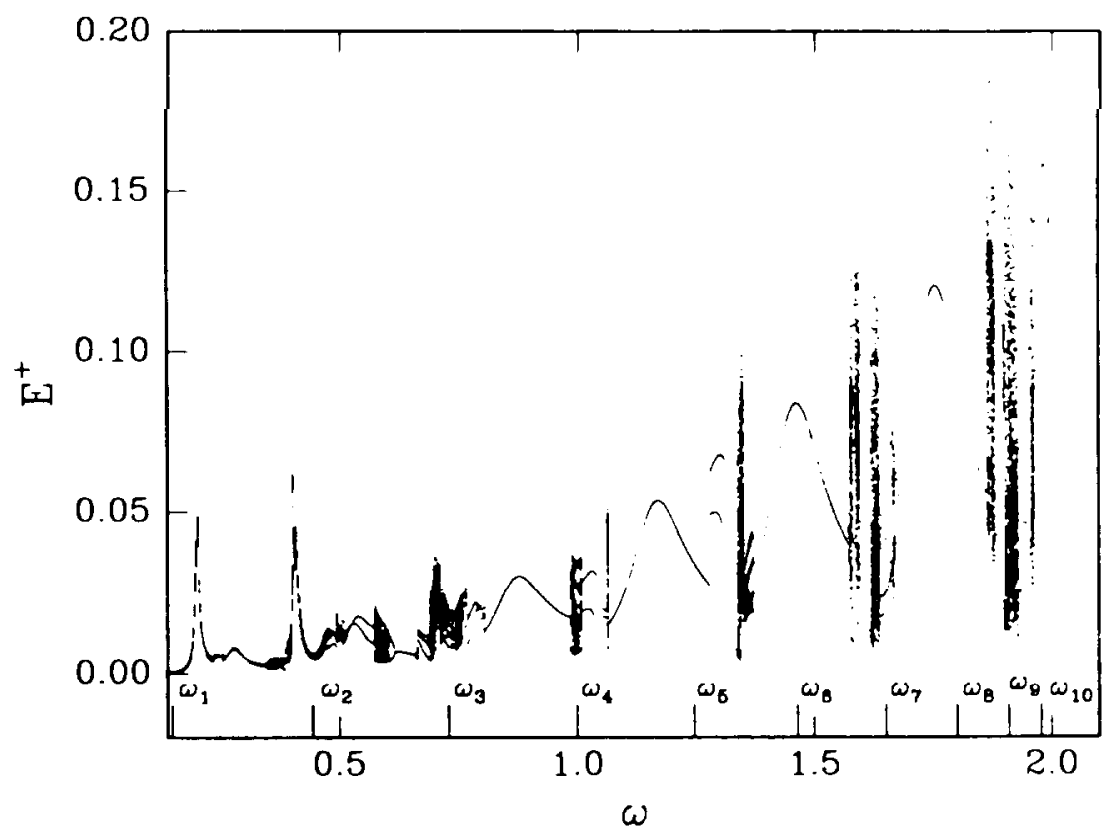

(b)

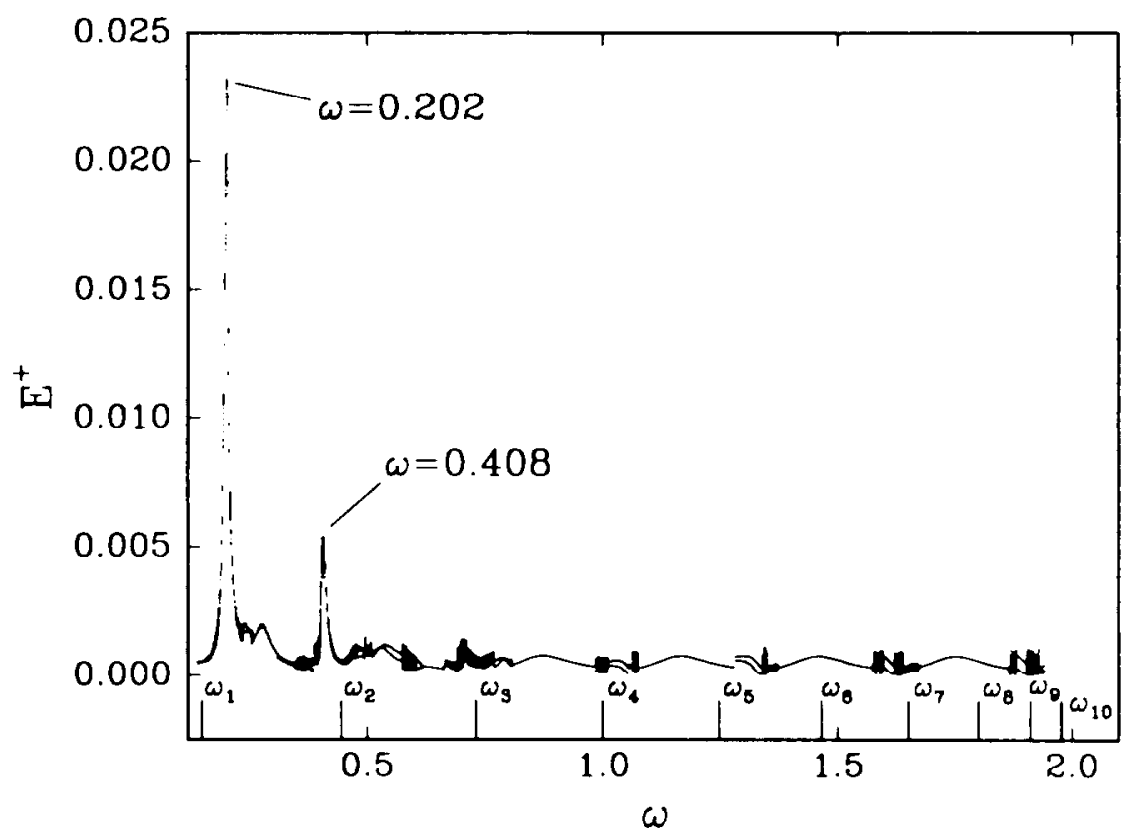

Fig. 6. Energy bifurcation diagrams: (a) same bifurcation diagram as Figs 4 and 5 (constant table amplitude), except plotted in terms of the system energy; (b) diagram done wih constant table velocity $\omega A=0.014$, same as the initial velocity amplitude for Fig. 6(a). The natural frequencies of the system between impacts are included for reference.

is shown in Figs 9 and 10. The impact Poincaré section of Fig. 10 (top) shows that the orbit contains a sequence of points in $\Sigma$ for which $v_{10} \rightarrow v_{\mathrm{T}}$, so, following the results of Section 3 , we conclude that an infinite number of impacts occurs in finite time. However, the timeand frequency-domain data of Fig. 9, as well as the stroboscopic section Fig. 10 (bottom), 

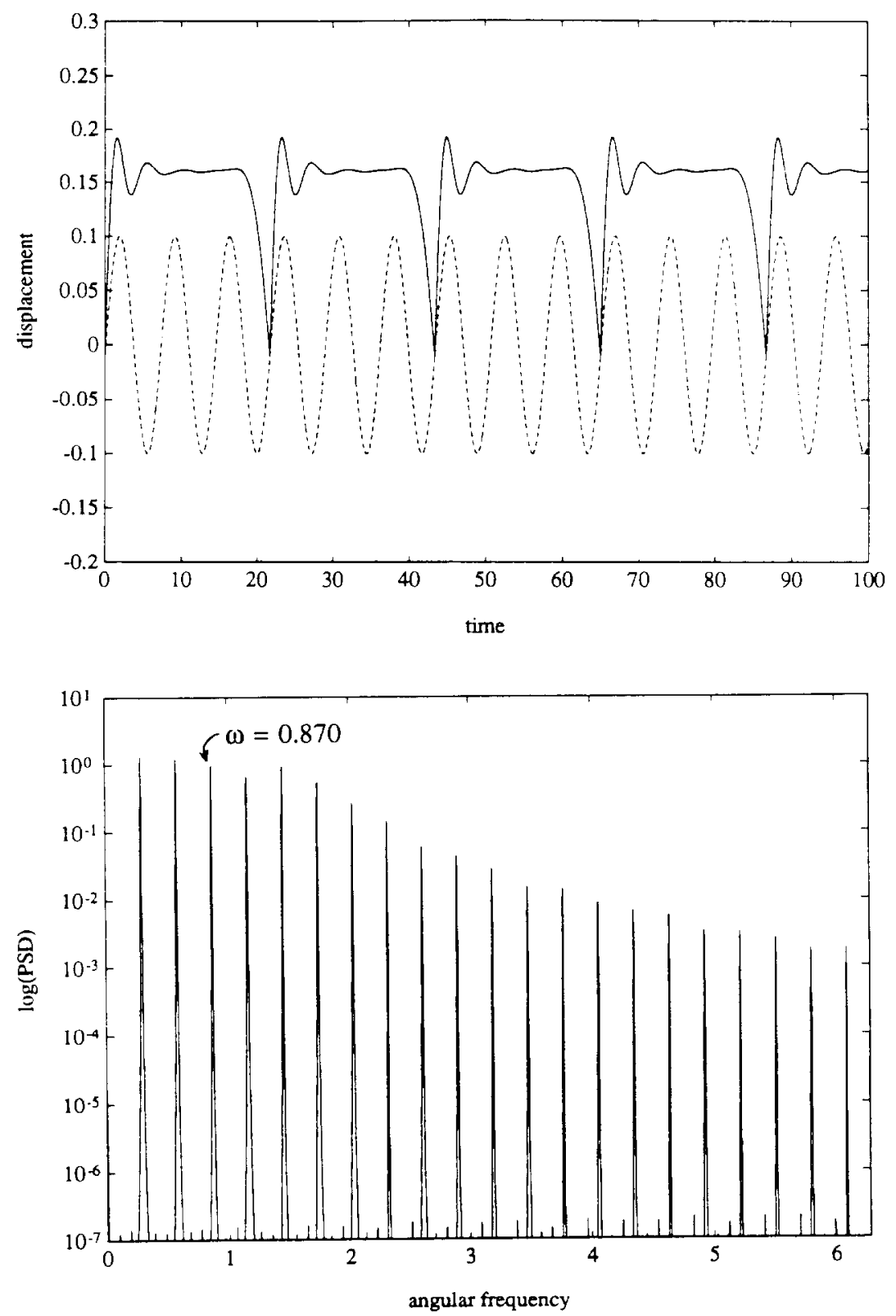

Fig. 7. A $P_{1,3}$ orbit $(\omega=0.870)$ : (top) time series $\left(x_{10}\right.$ is solid, table $x_{\mathrm{T}}$ is dashed); (bottom) power spectrum.

show that the orbit is period-4 with respect to the table motion. We therefore conclude that this is a $P_{x, 4}$ orbit: this seemingly paradoxical result is due to the intrinsic degeneracy of impacting systems, and can only be modelled with the system of equations (11) and (12) if they are modified as in our algorithm to pass through sticking events. Other $P_{x, n}$ orbits can be found in a similar manner. Finally, a typical chaotic orbit is displayed in Figs 11 and 12 . The power spectrum is, as one expects, broad-band in character, and a complicated attractor is visible in the sections of Fig. 12. Lyapunov exponents are not currently implemented in this simulation, however several of the chaotic orbits where checked 

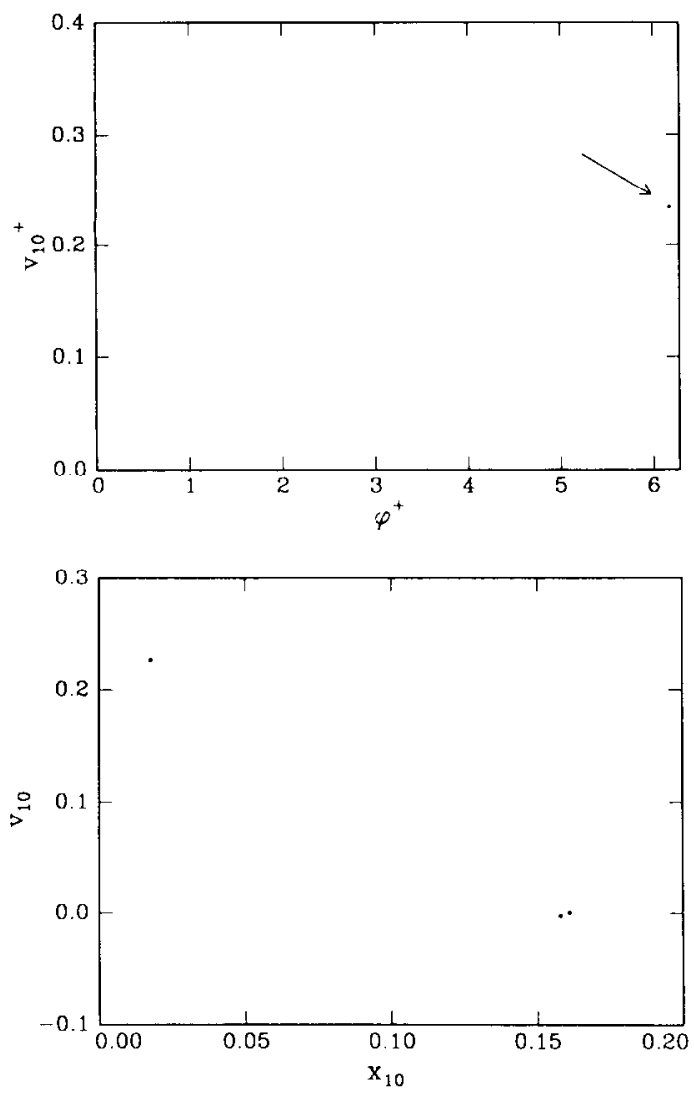

Fig. 8. Impact (top) and zero-phase stroboscopic (bottom) Poincaré sections of the orbit shown in Fig. 7. In this and other figures, $\phi=\bmod (\omega t, 2 \pi)$ is the table phase.

directly for sensitive dependance on initial conditions: results obtained with initial data with components differing by $10^{-8}$ were compared, and, as expected, the periodic orbits showed no change, but chaotic orbits diverged after about 100 impacts.

\section{DIMENSIONALITY AND SPATIAL COHERENCE}

That the dynamics of spatially continuous systems may be described by the evolution along a low-dimensional attractor is a primitive concept of the 'dynamical systems' methodology. The development of fractal dimension theory and, in particular, of the correlation dimension algorithm [16], has allowed dimensionality to be studied in physical and numerical experiments on a variety of fluid- and solid-mechanical systems (numerous examples can be found in the book by Moon [17], and in the references therein). From our point of view, the utility of fractal dimension estimates lies in the following: given a motion on an attractor with fractal dimension $d$, the number of phase space dimensions $m$ needed to contain the attractor is bounded by

$$
[d] \leqslant m \leqslant 2[d]+1 \text {, }
$$

where $[a]$ denotes the next greatest integer to $a$. The upper bound is due to Mañe's theorem [18], which states that, "typical" coordinatizations of a phase space containing an 

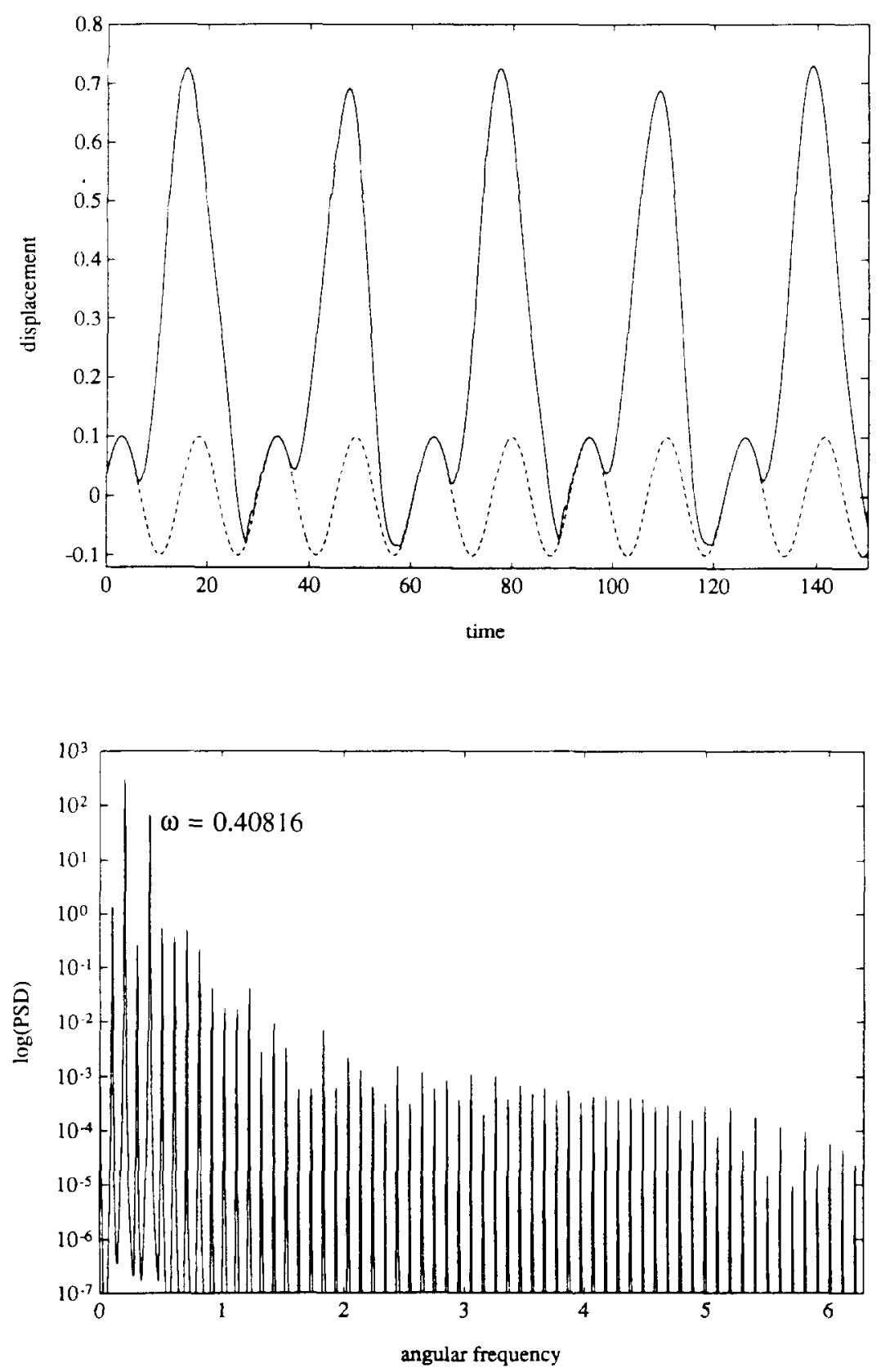

Fig. 9. A $P_{x, 4}$ orbit $(a=0.40816)$ : (top) time series $\left(x_{10}\right.$ is solid. table $x_{1}$ is dashed): (bottom) power spectrum.

attractor of dimension $d$ will leave the attractor lying in a subspace of dimension no greater than $2[d]+1$. Specifically, Mañé's theorem states that the coordinatizations for which this bound is true are residual in the space of all coordinatizations: however, while residual sets are typical (i.e. generic) in a topological sense, the results we present below demonstrate that this theorem may not be as definitive as it first appears. The application of dimension theory also suffers from several other limitations: there are no a priori error estimates for 

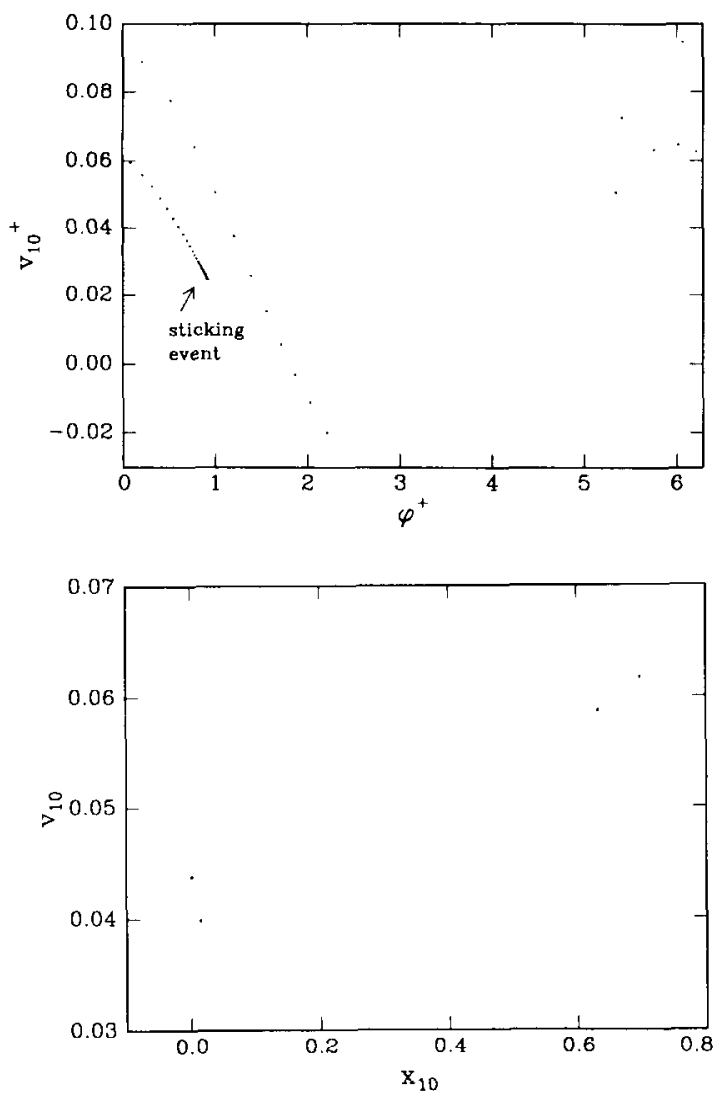

Fig. 10. Impact (top) and zero-phase stroboscopic (bottom) Poincaré sections of the orbit shown in Fig. 9.

the calculation of $d$; the estimate is sensitive to noise; and large amounts of data are required. Furthermore, the fractal dimension gives bounds on the number of degrees of freedom, but it says nothing about what the appropriate configuration variables should be. In particular, it does not yield any spatial information concerning important 'shape functions' or 'modes' in the system.

One method for studying dimensionality that promises to overcome some of these limitations is based on the proper orthogonal decomposition of the covariance of a random function [19], applied originally in mechanics to the study of turbulent fluid flows [20-23]. The object of study in the proper orthogonal decomposition method is the spatial correlation given by the dyadic product:

$$
\mathbf{R}\left(s, s^{\prime}\right)=\left\langle x(s, t) x\left(s^{\prime}, t\right)\right\rangle,
$$

where $\boldsymbol{x}$ is an appropriate field variable in space $s$ and time $t$ (such as displacement in solid mechanics, or velocity in fluid mechanics), and the brackets \langle\rangle denote the time average.

In our case, the spatial correlation is the tensor given by:

$$
\mathbf{R}=\langle\mathbf{x}(t) \mathbf{x}(t)\rangle=\left\langle x_{i}(t) x_{j}(t) \hat{\mathbf{e}}_{i} \hat{\mathbf{e}}_{j}\right\rangle=\left\langle x_{i}(t) x_{j}(t)\right\rangle \hat{\mathbf{e}}_{i} \hat{\mathbf{e}}_{j} \equiv R_{i j} \hat{\mathbf{e}}_{i} \hat{\mathbf{e}}_{i},
$$

where $x_{i}(t)$ is the displacement of the $i$ th mass with respect to the standard basis. Clearly, $\mathbf{R}$ is an Hermitian tensor, and thus its eigenvectors form a complete orthogonal set. Denoting the eigenvectors of $\mathbf{R}$ (called proper orthogonal modes) by $\hat{\boldsymbol{\phi}}_{i}$ and expanding the 

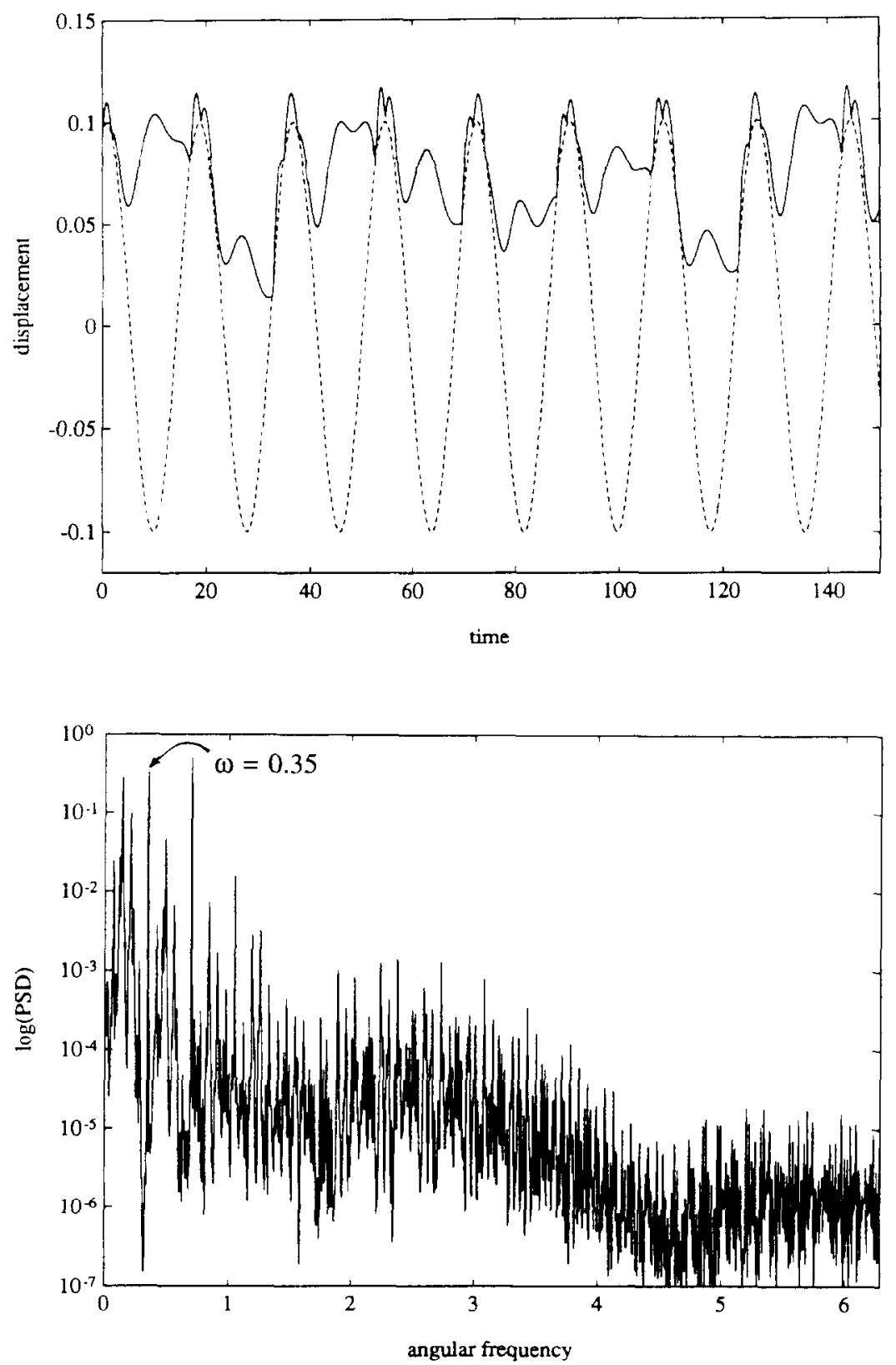

Fig. 11. A chaotic orbit $(\omega=0.35)$ : (top) time series $\left(x_{11}\right.$ is solid, table $x_{\mathrm{T}}$ is dashed); (bottom) power spectrum.

configuration vector $\mathbf{x}(t)$ as $\mathbf{x}(t)=\sum \alpha_{i}(t) \hat{\boldsymbol{\phi}}_{i}$, one has $\mathbf{R}=\left\langle\alpha_{i}(t) \alpha_{i}(t) \hat{\boldsymbol{\phi}}_{i} \hat{\boldsymbol{\phi}}_{j}\right.$. Since the $\hat{\boldsymbol{\phi}}_{i}$ are eigenvectors of $\mathbf{R}$, this implies that

$$
\hat{\boldsymbol{\phi}}_{i} \cdot \mathbf{R} \hat{\boldsymbol{\phi}}_{j}=\left\langle\alpha_{i}(t) \alpha_{j}(t)\right\rangle=\hat{\boldsymbol{\phi}}_{i} \cdot \lambda_{i} \hat{\boldsymbol{\phi}}_{j}=\lambda_{i} \delta_{i j} \text {. }
$$

The meaning of the proper orthogonal modes is thus clear: the modal amplitudes are linearly uncorrelated, and the associated eigenvalues are simply the mean-square modal amplitude (or power). Indeed, it can be shown that the eigenvectors of $\mathbf{R}$ give mode shapes 

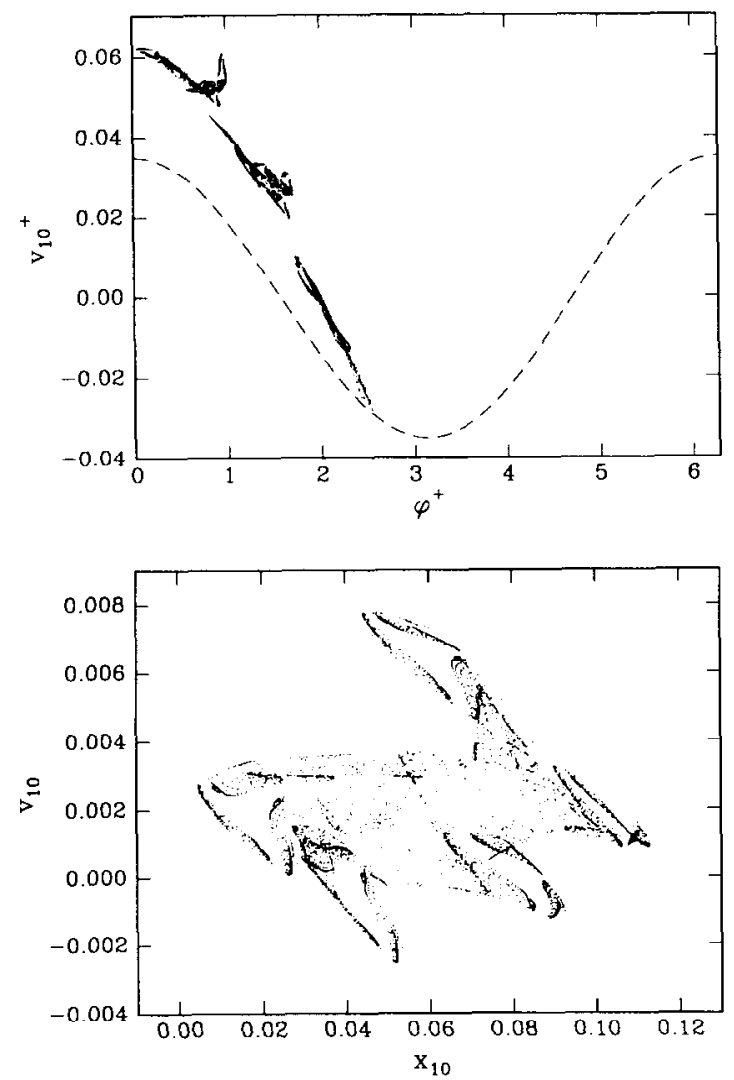

Fig. 12. Impact (top) and zero-phase stroboscopic (bottom) Poincaré sections of the orbit shown in Fig. 11.

that are optimal in a least squares sense: they capture more power per mode than any other set of basis functions. (We remark that this expansion is also known as the Karhunen-Loève decomposition [24].) The mean-square amplitude $P_{\mathrm{x}}$ (or 'signal power') of any motion is

$$
P_{\mathbf{x}}=\langle\mathbf{x}(t) \cdot \mathbf{x}(t)\rangle=\sum_{i=1}^{10}\left\langle x_{i}(t)^{2}\right\rangle=\sum_{i=1}^{10}\left\langle\alpha_{i}(t)^{2}\right\rangle=\sum_{i=1}^{10} \lambda_{i} .
$$

The optimality of the proper orthogonal modes therefore allows one to estimate the effective number of degrees of freedom excited in given motion by counting the minimum number of modes $N$ such that

$$
\sum_{i=1}^{N} \lambda_{i} \geqslant c P_{\mathbf{x}}
$$

for some fraction $c$ of the total power.

We now use the proper orthogonal decomposition to explore the relation between dimensionality, and spatial and temporal complexity in our impacting system. To apply the method, we take $c=0.99$, and calculate the correlation matrix $R_{i j}$ for various motions. The correlation matrix $R_{i j}$ was computed using $2 \times 10^{4}$ values of the configuration $\mathbf{x}(t)$ sampled at uniform intervals from the continuous-time response: the sampling rate chosen was in all cases roughly equal to the Nyquist frequency, although the results are not sensitive to this 
value. For each $R_{i j}$, an error matrix $E_{i j}$ was also computed using the standard deviation from the mean for each component of $R_{i j}$. The singular value decomposition was used to find the proper orthogonal modes $\hat{\phi}_{i}$ and the mean-square modal amplitudes $\lambda_{i}$. The largest singular value of $E_{i j}$ gives an upper bound on the error in the $\lambda_{i}$, which was used to ensure that sufficient data was taken so that the first $N \lambda_{i}$ used in the calculations where above the computational noise floor.

The $P_{x, m}$ orbit of Figs 9 and $10(\omega=0.40816)$ furnishes an example of a nontrivial, yet spatially coherent, motion. A portion of the evolution of the system in space and time is shown in Fig. 13. In Fig. 14 we show the first four proper orthogonal modes, and compare them to the normal modes for the system between impacts. The closeness of the proper orthogonal modes to the normal modes was typical of motions at all parameter values studied for this work, and suggests a simple interpretation of the cumulative statistical effect of repeated impacts: the spatial structure of the motion is close to what one would obtain by averaging over many impulse-response tests on the linear system. It must be emphasized, however, that the impacting system is strongly nonlinear (it is not linear even in the limit of "small oscillations"). In Fig. 15, the cumulative power is plotted: one sees that only two proper orthogonal modes are needed to capture over $99 \%$ of the total power. We thus estimate that only 2 degrees of freedom are excited for this attractor. The correlation dimension $d_{C}$ was calculated using the Grassberger-Procaccia algorithm on a data set consisting of $10^{4}$ points collected in the 20 -dimensional impact Poincaré section. For this and all other dimension estimates, the minimum absolute length scale considered valid was on the order of $10^{-4}$, consistent with the cumulative accuracy of the time-step calculations: a characteristic 'knee' was observed on the correlation integral curves at this scale, indicating that scales below this level were in the system's noise floor. For the $P_{\infty, m}$ data, it was found that $d_{\mathrm{C}}=1.23 \pm 0.01$ over a scaling range of better than $1000: 1$. Thus, the $P_{\infty, m}$ orbit offers an example of a nonchaotic fractal attractor. Dimension theory [inequality (19)] predicts that the dimension $m$ of the phase space is bounded by 2 and 5 . which is consistent with the estimate of 2 proper orthogonal modes (2 phase-space dimensions per mode plus time yields a phase space with dimension 5).

Figure 16(a) shows the spatial evolution for the chaotic orbit of Figs 11 and 12 $(\omega=0.35)$. Though somewhat more complex spatially than the orbit of Fig. 13, a high degree of spatial coherence is still visible. Indeed, one finds that only 3 proper orthogonal modes are required to exceed $99 \%$ of the total power. This is entirely consistent with the correlation dimension estimate of $d_{C}=2.21 \pm 0.04$ over a 20:1 scaling range: dimension theory predicts a phase space dimension of between 3 and 7 , and 3 modes plus time meet

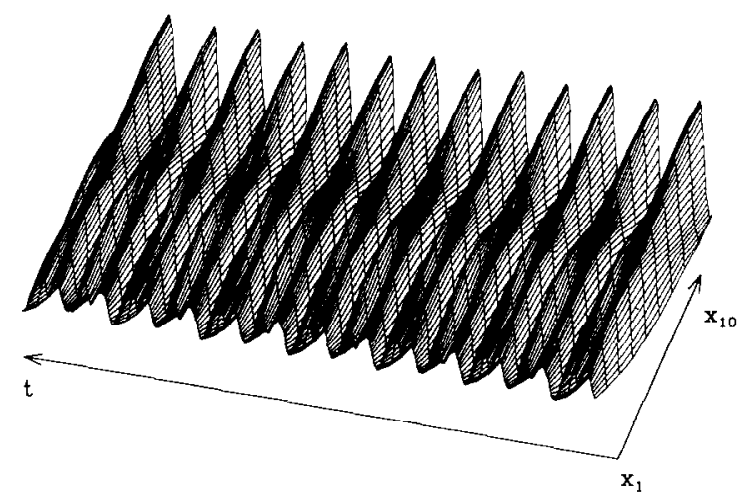

Fig. 13. Spatial evolution of the $P_{x .4}$ orbit of Figs 9 and $10(2000$ time steps with $\Delta t=0.2)$. 

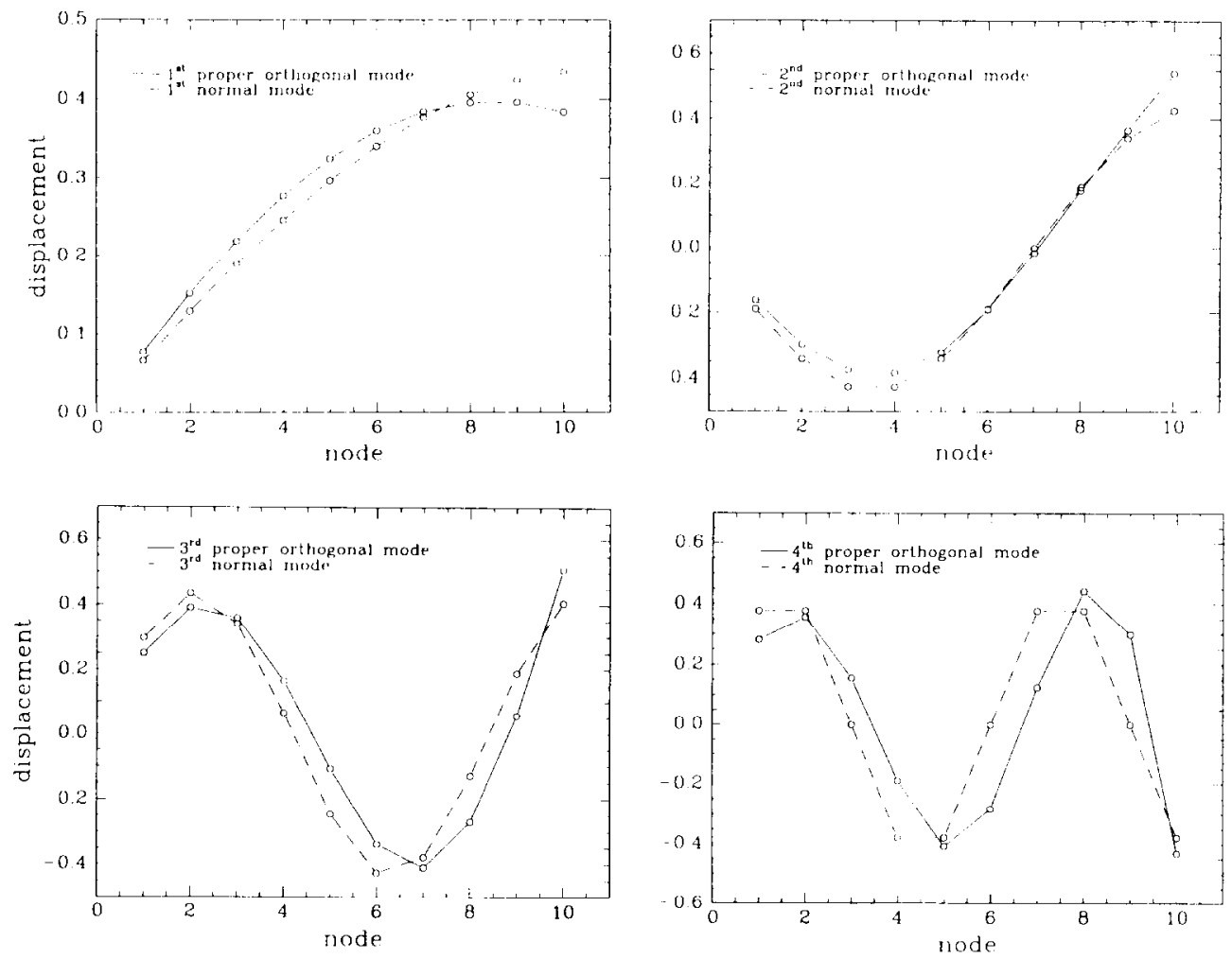

Fig. 14. Comparison between first four proper orthogonal modes and the first four normal modes of the non-impacting linear system $(\omega=0.40816)$.

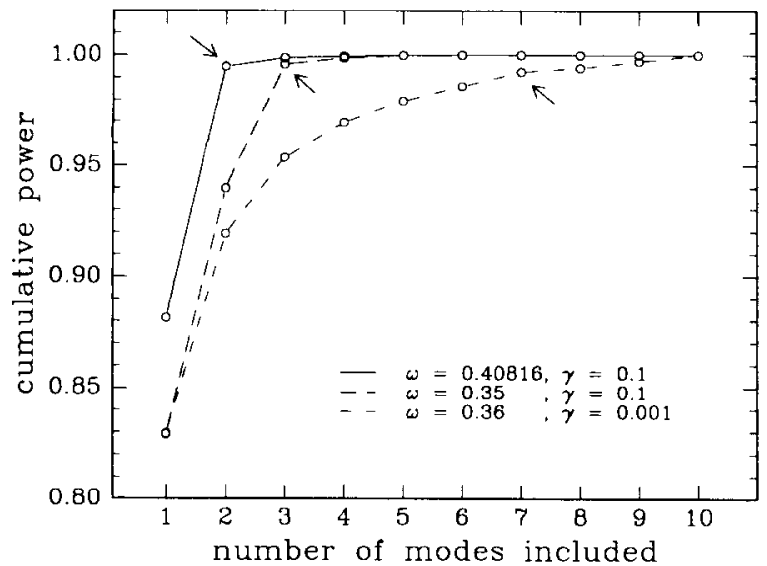

Fig. 15. Cumulative power in first $n$ proper orthogonal modes for several cases. Arrows mark number at which cumulative power exceeds $99 \%$ of total (refer also to Table 1 ).

the upper bound exaclly. A more strongly chaotic case is shown in Fig. 16(b), for which $\omega=0.36$ and the damping parameter has been lowered to $\gamma=0.01$. The visually apparent spatial complexity is confirmed by the fact that 5 proper orthogonal modes are required to exceed $99 \%$ of the total power, and by the result $d_{C}=6.2 \pm 0.2$ (over a $2.2: 1$ scaling 
range). Five modes plus time give a phase space of dimension 11 , which falls in the range predicted by dimension theory of 7 to 15 .

Table 1 displays additional information for these and other cases. The number of proper orthogonal modes $N$ needed to exceed $99 \%$ of the total power is shown, along with the resulting estimate of the phase space dimension, $2 N+1$. These estimates should be compared to the bounds (19) obtained with the correlation dimension. Also included in the
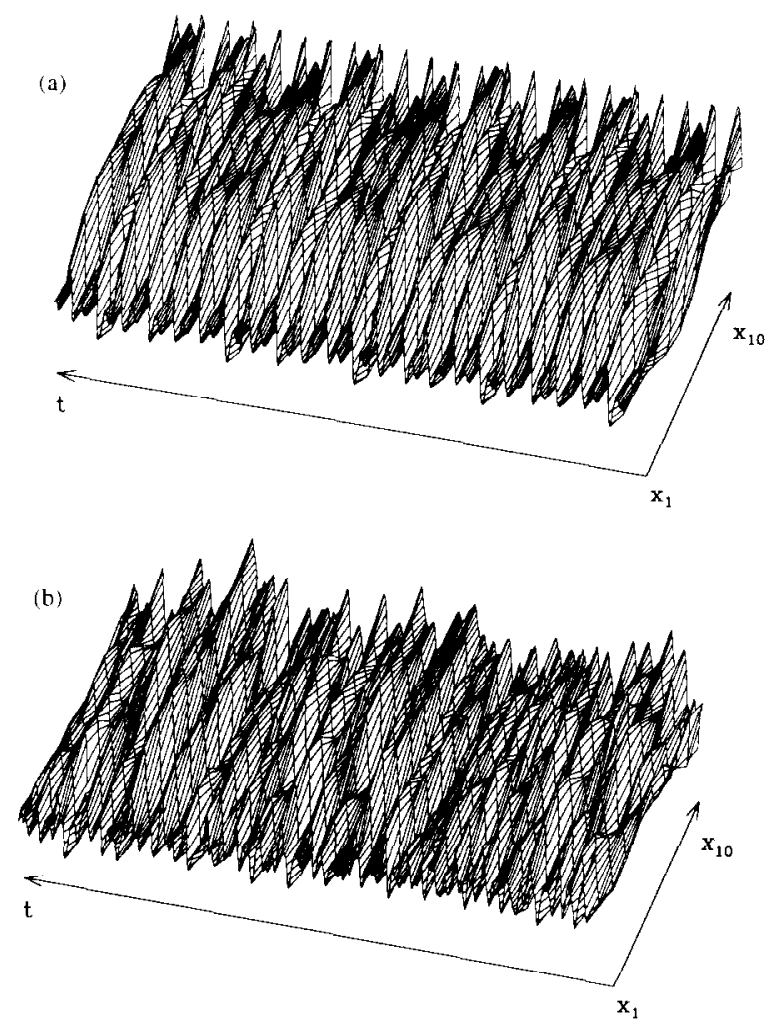

Fig. 16. Spatial evolution of chaotic orbits: (a) $\omega=0.35, \gamma=0.1 ;$ (b) $\omega=0.36, \gamma=0.01$ (each has 2000 time steps with $\Delta t=0.2)$.

Table 1. Summary of dimension and proper orthogonal decomposition results

\begin{tabular}{|c|c|c|c|c|c|c|c|c|}
\hline$\omega$ & $\gamma$ & $\begin{array}{c}\text { Type of } \\
\text { orbit }\end{array}$ & $N$ & $2 N+1$ & $d_{\mathrm{C}}$ & {$\left[d_{c}\right]$} & $2\left[d_{C}\right]+1$ & $r_{\max }: r_{\min }$ \\
\hline 0.34 & 0.1 & $P_{5.2}$ & 3 & 7 & $1.00 \pm 0.00$ & 1 & 3 & $220: 1$ \\
\hline 0.35 & 0.1 & chaotic & 3 & 7 & $2.21 \pm 0.04$ & 3 & 7 & $20: 1$ \\
\hline 0.36 & 0.1 & $P_{74.32}$ & 3 & 7 & $1.00 \pm 0.00$ & 1 & 3 & $3.3: 1$ \\
\hline 0.36 & 0.01 & chaotic & 5 & 11 & $6.2 \pm 0.2$ & 7 & 15 & $2.2: 1$ \\
\hline 0.36 & 0.001 & chaotic & 7 & 15 & $9.0 \pm 0.8$ & $9-10$ & $19-21$ & $1.8: 1$ \\
\hline 0.40816 & 0.1 & $P_{x \rightarrow+}$ & 2 & 5 & $1.23 \pm 0.01$ & 2 & 5 & $1000: 1$ \\
\hline 0.870 & 0.1 & $P_{1.3}$ & 5 & 11 & $1.00^{*}$ & 1 & 3 & $-*$ \\
\hline 1.346 & 0.1 & chaotic & 4 & $y$ & $2.58 \pm 0.05$ & 3 & 7 & $6.7: 1$ \\
\hline 1.750 & 0.1 & $P_{1,6}$ & 5 & 11 & $1.00^{*}$ & 1 & 3 & $-*$ \\
\hline 1.916 & 0.1 & chaotic & 3 & 7 & $2.9 \pm 0.1$ & 3 & 7 & $2.7: 1$ \\
\hline
\end{tabular}

* No error estimates or scaling regions are presented for these frequencies since the impact Poincaré section contained only one cluster of points, and hence all length scales were in the noise floor. 
table is the size of the scaling region associated with each dimension estimate. The table clearly shows that dimension theory as summarized by inequality (19) performs reasonably well, especially for the chaotic cases, but fails, sometimes in spectacular fashion, for some of the periodic motions. Some insight into how this can happen can be found by examining Fig. 17, in which the spatial evolution of the $P_{1.3}$ response with $\omega=0.870$ is shown. The sharp edges visible in the diagram, which indicate that the masses are being rapidly accelerated, make it necessary to include more proper orthogonal modes than required on the basis of purely topological arguments.

\section{SUMMARY AND DISCUSSION}

In this paper we have presented a numerical study of bifurcation, dimensionality, and spatial coherence in a 10 degree of freedom impact oscillator. Bifurcation diagrams for the system were obtained over the entire range of natural frequencies of the non-impacting system. Several characteristics of the diagrams merit further investigation. We have demonstrated, via relations (17) and (18), and in Fig. 6(b), that the input to the system is controlled by the table velocity. The energy bifurcation diagrams of Fig. 6 show two nonlinear resonances which do not occur at non-impacting natural frequencies: nor are the resonances related to an obvious, simple combination resonance. We remark that the resonance at $\omega \approx 0.202$ corresponds to a $P_{x, 1}$ sticking motion, and the resonance at $\omega=0.408$ corresponds to the $P_{\infty, 4}$ sticking motion of Figs 9, 10, and 13. However, one should not conclude that all sticking periodic orbits lead to similar resonances, since several others exist at higher frequencies away from the resonances. Work aimed at a more precise understanding of this phenomenon is currently being carried out.

Another feature of the bifurcation diagrams that requires further study is the banded pattern at higher frequencies in which a $P_{1, n}$ motion is alternated with a band of more complex responses (which include various $P_{n . m}$ orbits as well as chaotic responses). Figure 6(b) shows that, for constant small input to the system, the energy level of the successive bands remains approximately constant. Again, this pattern does not have an obvious relation to the natural frequencies of the non-impacting system.

We have applied the proper orthogonal decomposition to study the relation between dimensionality and spatial structure for the system. A simple, total power criterion was used to estimate the number of active degrees of freedom for various motions (the results are summarized in Table 1). Clearly, taking the cutoff to be $99 \%$ of the total power is

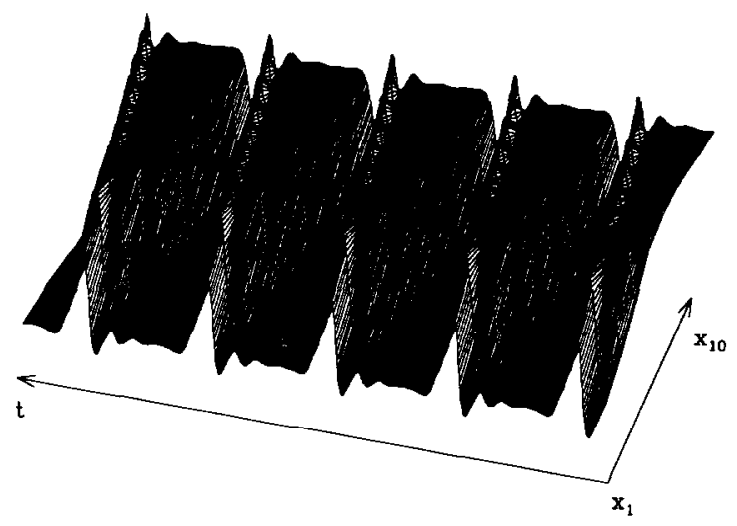

Fig. 17. Spatial evolution of the $P_{1,3}$ orbit with $\omega=0.870(2000$ time steps with $\Delta t=0.1)$. 
somewhat arbitrary, and a more objective criterion would be preferable. Nevertheless, this simple method allowed us to examine the predictions of dimension theory in a consistent fashion: in five of the ten cases examined, the estimated number of phase-space dimensions $(2 N+1)$ fell within the bounds predicted by inequalities (19). Four of these five were chaotic orbits (with $\omega$ equal to $0.35,0.36,0.36$, and 1.916), and even the remaining chaotic orbit (with $\omega=1.346$ ) was reasonably close to the predicted bounds. The remaining result that fell within the predicted bounds was for a $P_{x, 4}$ sticking periodic orbit. The more regular orbits, however, failed to meet the predictions of dimension theory, with the poorest results belonging to the orbits which were simplest (in a topological sense): the $P_{1,3}$ and $P_{1,6}$ orbits for $\omega$ equal to 0.870 and 1.750 , respectively. It is clear that rapid accelerations in such motions (which lead to the 'sharp edges' in the spatial evolution diagram of Fig. 17) necessitate the inclusion of more modes in any expansion: it may be that chaotic orbits tend to 'smooth out' such edges, thus explaining the better match with the predictions of dimension theory. At a more fundamental mechanical level, the singular nature of the impact condition tends to make the system dimensionally nonhomogeneous with respect to time: one should expect more modes to be excited 'just after' impact than on average. The uniform, continuous time average we did to obtain our proper orthogonal modes clearly has a big influence on the nature of the results. In contrast, preliminary work indicates that, as expected, averaging over impact Poincaré section data, rather than continuous-time data, significantly increases the number of modes required. Current work is aimed at understanding the importance to the proper orthogonal decomposition method of such factors as sampling method, modal damping values, and impact-time statistics.

We have found that the spatial structure, as evidenced by the proper orthogonal modes, is, in this case, virtually identical to that predicted by examination of the normal modes for the non-impacting system. This implies that the statistical effect of repeated impacts on the spatial structure of the nonlinear system is equivalent to what one would obtain by averaging over an ensemble of impulse response tests of the non-impacting linear system. Furthermore, this statistical equivalence does not appear to depend strongly on the type of response of the impacting system (in fact, we found much closer correspondence between the proper orthogonal modes and the normal modes for strongly chaotic responses). This suggests that deliberate excitation of chaotic impacting responses may be of practical use for engineering modal analysis. Of course, the proper orthogonal decomposition method is more general than standard modal analysis methods, since it makes no reference to linearity: being entirely based on statistics, it will yield useful information even when the response between impacts is nonlinear.

Acknowledgements - This work was supported by the National Science Foundation grant MSS 89-10814, and by the Air Force Office of Scientific Research grant AFOSR-91-0430.

\section{REFERENCES}

1. P. J. Holmes, The dynamics of repeated impacts with a sinusoidally vibrating table, J. Sound Vihr. 84(2). $173-189$ (1982).

2. S. W. Shaw, The dynamics of a harmonically excited system having rigid amplitude constraints. part I: Subharmonic motions and local bifurcations, ASME J.Appl. Mech. 52, 453-458 (1985).

3. S. W. Shaw, The dynamics of a harmonically excited system having rigid amplitude constraints, part II: Chaotic motions and global bifurcations, ASME I. Appl. Mech. 52, 459-464 (1985).

4. R. M. Everson. Chaotic dynamics of a bouncing ball, Physica 19D, 355-383 (1986).

5. G. S. Whiston, Global dynamics of a vibro-impacting linear oscillator. J. Sound Vibr. 118. 395-429 (1987).

6. G. S. Whiston. Singularities in vibro-impact dynamics. J. Sound Vibr. 152(3), 427-460 (1992).

7. F. C. Moon and S. W. Shaw. Chaotic vibration of a beam with nonlinear boundary conditions. Int. I. Non-Linear Mech. 18, 465-477 (1983).

8. N. B. Tufillaro and A. M. Albano, Chaotic dynamies of a bouncing ball, Am. J. Phys. 54(10). 939-1)44 (1986). 
9. N. B. Tufillaro, T. M. Mello, Y. M. Choi and A. M. Albano, Period doubling boundaries of a bouncing ball, J. de Physique 47, 1477-1482 (1986).

10. T. M. Mello and N. B. Tufillaro, Strange attractors of a bouncing ball, Am. J. Phys, 55(4), (1987).

11. Z. J. Kowalik, M. Franaszek and P. Pierański, Self-reanimating chaos in the bouncing-ball system, Phys. Rev. A 37(10), 4016-4022 (1988).

12. F. Hendriks, Bounce and chaotic motion in print hammers, IBM J. Res. Dev. 27(3), 273-280 (1983).

13. W. H. Press, B. P. Flannery, S. A. Teukolsky and W. T. Vetterling, Numerical Recipes: The Art of Scientific Computing. Cambridge University Press, New York (1986).

14. D. T. Greenwood, Principles of Dynamics, 2nd Edn. Prentice-Hall, Englewood Cliffs, NJ (1988).

15. K. Geist and W. Lauderborn, The nonlinear dynamics of the damped and driven Toda chain, Physica 31D, $103-116(1988)$.

16. P. Grassberger, and I. Proccacia, Characterization of strange attractors, Phy. Rev. Lett. 50, 346-349 (1983).

17. F. C. Moon, Chaotic and Fractal Dynamics: An Introduction for Applied Scientists and Engineers. Wiley, New York (1992).

18. R. Mañé, On the dimension of the compact invariant sets of certain nonlinear maps, in Dynamical Systems and Turbulence, edited by D. A. Rand and L.-S. Young, Springer Lecture Notes in Mathematics, 898, 230-242 (1988).

19. M. Loève, Probability Theory, Van Nostrand, Princeton, NJ (1963).

20. J. L. Lumley, The structure of inhomogencous turbulcnt flows, in Amospheric Turbulence and Radio Wave Propagation, edited by A. M. Yaglom and V. I. Tatarski, pp. 166-178. Nauka, Moscow (1967).

21. L. Sirovich, Turbulence and Dynamics of coherent structures, part I: coherent structures, Quart. Appl. Math. 45, $561-571$ (1987).

22. L. Sirovich, Turbulence and dynamics of coherent structures, part II: symmetries and transformations, Quart. Appl. Math. $45573-582$ (1987).

23. I.. Sirovich. Turhulence and dynamics of coherent structures, part III: dynamics and scaling, Quart. Appl. Math. 45, 583-590 (1987).

24. R. G. Ghanem and P. D. Spanos, Stochastic Finite Elements: A Spectral Approach. Springer, New York (1991).

\section{APPENDIX}

When the system of Fig. 1 is between impacts, it is governed by equations (1) and (2). The natural frequencies of the non-impacting system are found by standard numerical means to be as shown in Table A1.

Table A1.

\begin{tabular}{lccc}
\hline $\begin{array}{l}\text { Mode } \\
\text { number } \\
n\end{array}$ & $\begin{array}{c}\text { Natural } \\
\text { frequency } \\
\omega_{n}\end{array}$ & $\begin{array}{c}\text { Mode } \\
\text { number } \\
n\end{array}$ & $\begin{array}{c}\text { Natural } \\
\text { frequency } \\
\omega_{n}\end{array}$ \\
\hline 1 & 0.150 & 6 & 1.466 \\
2 & 0.445 & 7 & 1.652 \\
3 & 0.731 & 8 & 1.802 \\
4 & 1.000 & 9 & 1.911 \\
5 & 1.247 & 10 & 1.978 \\
\hline
\end{tabular}

